\title{
Shared strategies for behavioral switching: understanding how locomotor patterns are turned on and off
}

\author{
Karen A. Mesce ${ }^{\text {* }}$ and Jonathan T. Pierce-Shimomura ${ }^{2}$ \\ Graduate Program in Neuroscience, Departments of Entomology and Neuroscience, University of Minnesota, Saint Paul, MN, USA \\ Section of Neurobiology, University of Texas, Austin, TX, USA
}

Edited by:

Kathleen A. French, University of

California San Diego, USA

Reviewed by:

Catharine H. Rankin, University of

British Columbia, Canada

Kevin Briggman, Max-Planck-Institute

for Medical Research, Germany

Kathleen A. French, University of

California San Diego, USA

*Correspondence:

Karen A. Mesce, Departments of

Entomology and Neuroscience,

University of Minnesota, 219 Hodson

Hall, 1980 Folwell Avenue, St. Paul, MN

55108, USA.

e-mail:mesce001@umn.edu
Animals frequently switch from one behavior to another, often to meet the demands of their changing environment or internal state. What factors control these behavioral switches and the selection of what to do or what not to do? To address these issues, we will focus on the locomotor behaviors of two distantly related "worms," the medicinal leech Hirudo verbana (clade Lophotrochozoa) and the nematode Caenorhabditis elegans (clade Ecdysozoa). Although the neural architecture and body morphology of these organisms are quite distinct, they appear to switch between different forms of locomotion by using similar strategies of decision-making. For example, information that distinguishes between liquid and more solid environments dictates whether an animal swims or crawls. In the leech, dopamine biases locomotor neural networks so that crawling is turned on and swimming is turned off. In C. elegans, dopamine may also promote crawling, a form of locomotion that has gained new attention.

Keywords: decision-making, medicinal leech, Caenorhabditis elegans, behavioral choice, dopamine

\section{INTRODUCTION}

Throughout our daily lives we frequently shift from one behavior to another. For example, while reading this article you might shift your attention to a knock on your office door or glance at your clock in anticipation of an important appointment. In fact, behavioral switches are an adaptive feature across a broad range of animal species and can maximize an organism's viability and differential reproductive success. Ultimately, switching between behaviors helps an animal to cope with its changing environment or internal states, and appropriate responses to these stimuli can be critical for survival.

Although behavioral switching and choice are inextricably linked, defining what constitutes behavioral choice and understanding how decisions are made remain topics of intense discussion and research (Kristan and Shaw, 1997; Körding, 2007; Kristan, 2008; Kiani and Shadlen, 2009). In this "Perspectives" article we highlight some of the elegant and conserved neural strategies that have been found to contribute to the selection of one form of locomotor behavior vs. another. These biologically crafted strategies exemplify ways in which the environment and internal state of an animal can generate behavioral switching. We will also argue that these mechanisms are found consistently across animal groups separated by millions of years of evolution. Our aim is to describe a collection of neurobiological control mechanisms that switch locomotor behaviors on or off. We will emphasize the importance of context for appropriate behavioral switching and will provide examples of sensorimotor strategies that are similar across taxa.

\section{BEHAVIORAL SWITCHES: SELECTING DIFFERENT FORMS OF LOCOMOTION}

Individual animals can change their form of locomotion or gait quickly. For instance, an animal may switch from walking to running or switch from crawling to swimming depending on the environmental conditions present. What external and internal events shape the switching from one form of locomotion to another?

Different locomotor patterns are often associated with different circumstances. These generally include: (1) behavioral states related to searching for food or a mate; escaping from predators; interacting during agonistic encounters, and (2) environmental sub-niches, such as rough vs. smooth terrain; water vs. land or air. During the course of a species' evolutionary route, rapid behavioral switching may have evolved to prevent inefficient movements during escape behaviors and/or in conjunction with changes in terrain. Once a neural mechanism evolved to switch the locomotor form, this trait potentially would be favored due to its efficiency in escape-related survival or the maximization of resource acquisition within differing niches. Although it may be potentially adaptive for an animal to use a variety of forms or gaits under different circumstances, it might be maladaptive to get "stuck" when switching between forms/gaits if the transition were inefficient. Therefore, we propose that during the evolution of a given transition, there must be selective pressure to favor effective behavioral switching to ensure that competing motor patterns are switched on and off in a precise manner.

\section{A TALE OF TWO WORMS}

Throughout the remainder of this article, we will focus on two distantly related organisms and the common biological mechanisms they use to switch efficiently between different forms of locomotion. The medicinal leech, Hirudo species, is a well-studied annelid model of neural circuits underlying locomotion and other behaviors (reviewed in Kristan et al., 2005). Successful electrophysiological analysis of neural circuits involved in quantifiable behaviors has been driven largely by the leech's simple body plan and 
size $(10 \mathrm{~cm})$, which allows convenient dissection of its uniquely identifiable neurons. The considerably smaller nematode worm (1 mm), Caenorhabditis elegans, is an established genetic model of behavior (de Bono and Maricq, 2005) and has afforded scientists the ability to manipulate neural circuits via genetic tools still unavailable for the leech. Modern molecular phylogenies (Dunn et al., 2008) show that these two species are members of two widely divergent clades of bilaterally symmetric animals. In fact, they are almost as distant from one another as either is from vertebrates. Despite their physiological and morphological differences (e.g., the leech is segmented and contains over 10,000 neurons as compared to the 302 neurons in C. elegans), a surprising number of shared control strategies for locomotion is now emerging.

The leech has two basic forms of locomotion, swimming and crawling, and each is easily distinguished from the other. Swimming consists of a rostrocaudal sinusoidal wave of body movements involving antiphasic contractions of dorsal and ventral muscles. Crawling entails the co-contractions of dorsal and ventral muscles, which enable the whole-body to elongate and contract. Swimming has a relatively short period between repeated cycles (0.3-1 s), whereas crawling has a much longer one (7-20 s) (Kristan et al., 2005; Puhl and Mesce, 2008). Understanding the neural bases for these behaviors has benefited from two powerful preparations: the "nearly-intact preparation", in which overt locomotion can be monitored while the activity of identified neurons is recorded in selected ganglia, and the "isolated nerve cord preparation" wherein fictive crawling and swimming behaviors are measured based on established patterns of neuronal output (Kristan et al., 2005). In both preparations, the leech's relatively large neurons $(25-100 \mu \mathrm{m}$ diameter) can be recorded simultaneously over extended periods (>3 h) with electrophysiological methods. Voltage-sensitive dyes have permitted the optical recording of hundreds of identified neurons simultaneously and are providing new insights into the cellular mechanisms of decision-making and multi-functional circuits (Briggman and Kristan, 2006, 2008). The nearly intact preparation is particularly useful because the goal of matching locomotor kinematics with the activity of identified neurons can be met.

In nearly intact leech preparations individual cephalic neurons were identified that either activated locomotion or were rhythmically active during locomotion (Esch et al., 2002; Mesce et al., 2008). Electrical excitation of a single neuron, named R3b-1 (bilaterally paired), was sufficient to elicit sustained locomotor activity (Esch et al., 2002). The form of locomotion induced, however, was entirely dependent on whether the body of the leech was suspended in fluid or allowed to lie upon a dryer surface (Esch et al., 2002). Thus depolarization of a single R3b-1 neuron resulted in a context-dependent response that was gated by sensory input from either an aquatic or terrestrial environment. Additional studies of overt crawling and swimming confirmed that leeches will quickly switch to a crawling mode when encountering shallow water (Esch et al., 2002; Puhl and Mesce, 2010). Furthermore, when fluid levels were ambiguous, a swim-crawl hybrid locomotor pattern emerged in both fully- and nearly intact animals (Esch et al., 2002). Thus the decision to locomote is hierarchical, made at the level of a single higher-order descending interneuron, but the switching (and selection in this case) of various forms of locomotion is based on the integration of contextual somatosensory cues. If descending signals from the cephalic ganglion are removed reversibly, the leech looses its ability to initiate crawling even in the presence of relevant sensory stimulation (Puhl and Mesce, 2010). Thus the decision to crawl is entirely dependent on cephalic descending input. The decision to swim, however, is not so dependent on cephalic inputs. For example, tactile body stimulation can reliably induce swimming when the cephalic ganglion is removed reversibly (Puhl and Mesce, 2010). Although the cephalic ganglion contains a variety of neurons that can gate or trigger swimming (Brodfuehrer and Friesen, 1986), downstream swim-gating neurons are also competent to activate the swim oscillators (Kristan et al., 2005). Last, the biomechanics of an animal clearly must constrain which locomotor behaviors are available for selection or the time of transition between forms; for example, such constraints may help to explain why leech swimming and crawling are always initiated with an elongation of the body and the excitation of similar (circular) muscles (Esch et al. 2002; Kristan et al., 2005).

The results above provide a striking example of how sensory information from the environment is fundamentally important for behavioral switching, but what about the internal state of the animal? Are there conditions under which the leech will be strongly biased to crawl or swim because of its internal state? Recent studies indicate that specific biogenic amines significantly bias the mode of locomotion available to an animal. For example, dopamine has been shown to turn on the underlying neural circuits generating leech crawling behavior (Puhl and Mesce, 2008, 2010). In addition, dopamine prevents swimming from becoming activated (Crisp and Mesce, 2004). In preparations in which fictive locomotion was recorded, dopamine blocked swimming even when the CNS was electrically stimulated in attempts to produce swimming (Crisp and Mesce, 2004). In the presence of dopamine, stimulation of cell R3b-1 was found to induce crawling but never swimming, regardless of the swim-specific contextual cues present (Puhl and Mesce, personal observations). Thus dopamine not only activates crawling, it prevents swimming as well, providing a clear example of a chemical switch for locomotor behavior. Although it is unknown how the levels of dopamine fluctuate normally within the leech, we can speculate that they might significantly rise when the leech comes in contact with its host. Typically, the leech switches to crawling at this time and some evidence suggests that dopamine is linked to feeding (biting) behavior (O'Gara et al., 1991). Because swimming often requires detachment of both its front and rear suckers, it makes sense that this form of locomotion needs to be suppressed to prevent loss of contact with the host.

Is there a neurohormone or modulator that switches on swimming in the leech? The answer to this question has been long known, and solid evidence indicates that serotonin is the key modulator for activating swimming (Willard, 1981; Friesen and Kristan, 2007). During active feeding behavior, however, serotonin was elegantly shown to serve as a direct and powerful inhibitor of the sensory gating for swimming, crawling, and other body movements (Gaudry and Kristan, 2009). It is noteworthy that the central activation of swimming was not inhibited. Because serotonin is known to activate the central swim oscillators, one might question how swimming becomes inactivated during feeding? Paradoxically, focal delivery of serotonin to the cephalic ganglion, but not the nerve cord, was found to suppress swimming by influencing the decision to swim (Crisp and Mesce, 2003). Bath application of serotonin was deemed a physiologically appropriate delivery protocol because it matched the anatomically 
based paracrine-like release of serotonin within the CNS (Crisp and Mesce, 2003). Furthermore, preliminary data support the idea that dopamine trumps serotonin: a mixture of serotonin and dopamine results in no swimming (Puhl and Mesce, personal observation). Thus as long as dopamine is present at high enough concentrations during feeding, serotonin's actions on the swim central pattern generator are observably blocked. Perhaps, when the levels of dopamine drop after a feeding session and serotonin levels are low enough to prevent sensory neuron pre-synaptic inhibition (Gaudry and Kristan, 2009), the leech is once again able to switch on its swimming behavior.

What parallels exist for the regulation of locomotion in C. elegans? In the leech, the dorsal-ventral undulations used in swimming are unambiguously distinct from the whole-body elongations and contractions made during vermiform crawling (Kristan et al., 2005; Puhl and Mesce, 2008). Only recently have several labs considered whether C. elegans also exhibits distinct crawling and swimming forms of motion (Korta et al., 2007; Pierce-Shimomura et al., 2008; Berri et al., 2009). Similar to swimming in the leech, both forms of motion in $C$. elegans appear to depend on thrust generated by bends passed along the body. Kinematic analysis revealed that animals crawl with a persistent S-shaped posture on a dry substrate with $\sim 1 \mathrm{~Hz}$ high-amplitude bends, and swim in liquid with $\sim 2 \mathrm{~Hz}$ lower amplitude bends (PierceShimomura et al., 2008). The two forms of motion also differ in the pattern of bend propagation: bends propagate at uniform velocity for crawling, but "whip" to the back of the body after characteristic pauses when the whole-body of the worm is bent into a C-shaped posture during swimming (Pierce-Shimomura et al., 2008).

In C. elegans and the leech, crawling and swimming are further distinguished by the selective overlay of searching-related motor patterns while crawling. For example, superimposed upon its $1 \mathrm{~Hz}$ dorsal-ventral crawl bends, C. elegans wiggles its "nose" at $\sim 10 \mathrm{~Hz}$ in all three dimensions. This behavior has been termed "foraging" because the worm seems to use it as a way to search for food or the detection of substrate properties. An analogous behavior, although considerably slower, is displayed during crawling in the leech (Gray et al., 1938; Cacciatore et al., 2000; Mesce et al., 2008). These exploratory-type behaviors are turned off during swimming in both the leech (Kristan et al., 2005) and worm (PierceShimomura et al., 2009; Vidal-Gadea et al., 2009).

The genetic tractability of C. elegans has allowed crawling and swimming to be distinguished with transgenic tools and mutation. By imaging calcium transients in muscles of freely moving animals, with the genetically encodable calcium-indicator Chameleon, the two forms of motion were shown to be subserved by distinct patterns of muscle activity (Pierce-Shimomura et al., 2008). In the future, newer imaging techniques may enable the activity patterns of identified neurons to be revealed in freely moving animals (Faumont et al., 2008; Ben Arous et al., 2010). Crawl and swim are also distinguished genetically in the worm because some mutations have a more severe effect on swimming than crawling (Miller et al., 1996; Pierce-Shimomura et al., 2008). The specific effects rendered by mutation may be thought of as being analogous to how a pharmacological agent may preferentially affect one form of locomotion over another form in other animals.

Although C. elegans crawls and swims with distinct kinematics, it remains controversial whether crawling and swimming represent distinct gait-like forms of motion or simply altered versions of a single form (Korta et al., 2007; Pierce-Shimomura et al., 2008; Berri et al., 2009). This issue is usually addressed in animals with limbs by studying how kinematics vary as an animal moves at different speeds on a treadmill. Gaits are indicated if the animal shows a discontinuous transition in kinematics with a continuous increase in speed just as humans show a sudden switch from walking to running at $\sim 4 \mathrm{~m} / \mathrm{s}$ (Alexander, 1989). In place of a treadmill, C. elegans researchers control speed by immersing the worm in different concentrations of viscous methylcellulose (Korta et al., 2007; Berri et al., 2009). Two studies have found that bend frequency decreases gradually with increased viscosity (Korta et al., 2007; Berri et al., 2009). An initial study by Korta et al. (2007) reported that that the worm unexpectedly and inexplicably maintained the same swim-like wavelength (C-shaped waveform) in both low and high viscosities, but this result has not been reproduced (Fang-Yen and Samuel, personal communication). A second study instead found that wavelength decreases gradually with increased viscosity (Berri et al., 2009). These studies suggest that crawling and swimming may represent a single form of motion in $C$. elegans. However, recent unpublished findings show that the worm spontaneously alternates between bouts of crawling and swimming in intermediate viscosity solutions (Pierce-Shimomura et al., 2009; Vidal-Gadea et al., 2009). This observation may have been missed when kinematic values were averaged in previous studies. This new result nicely complements how sensory mutants move in liquid. Mutations that specifically perturb the development and function of all sensory cilia cause a peculiar defect in swimming - these mutants switch spontaneously between crawl and swim forms of motion in liquid (Pierce-Shimomura et al., 2008). Together, these data suggest that the switch between crawling and swimming reflects a "decision" made by the worm based on sensory input just as it does in the leech.

While the neural basis for crawling and swimming has been studied for decades in the leech, how $C$. elegans switches between crawl and swim is just beginning to be investigated. Taking a hint from the leech, the Pierce-Shimomura lab is investigating whether dopamine plays a role in locomotor switching. Preliminary results suggest that dopamine is necessary and sufficient to facilitate a gaitlike switch from swim to crawl in C. elegans (Pierce-Shimomura et al., 2009; Vidal-Gadea et al., 2009). Interestingly, dopamine does not appear to be necessary to maintain the gross kinematics for either form of motion. Knock-out of the dopamine transporter gene, however, results in a gradual paralysis during swimming that depends on endogenous dopamine synthesis and a D2-type dopamine receptor (McDonald et al., 2007). Based on these results, this swim-induced paralysis might be explained by dopamine levels rising above a concentration that permits swimming. C. elegans has eight dopamine neurons that appear to act as mechanoreceptors sensitive to substrate texture (Sulston et al. 1975; Sawin et al., 2000). With potential parallels to the leech, dopamine neurons in $C$. elegans may become activated upon firm contact of the body with the substrate to trigger a switch from swimming to crawling.

How does C. elegans switch from crawl to swim? While serotonin has a known role in slowing the crawling speed of starved animals after finding food (Sawin et al., 2000), the Pierce-Shimomura lab has only begun to study whether serotonin has a role in swimming (Vidal-Gadea and Pierce-Shimomura, unpublished observations).An unbiased genetic screen for mutations that disrupt the crawl to swim transition revealed a key role for the newly described sodium-leak 
channel pathway (Pierce-Shimomura et al., 2008). These mutants are capable of crawling, but become frozen in posture within a fraction of a second upon entering liquid. The sodium-leak channels are widely expressed in the nervous system, so it remains unclear whether they facilitate the crawl to swim switch through neuromodulatory signaling, independent mechanisms, or both (Pierce-Shimomura et al., 2008). Recent work by Lu et al. (2009) has determined that the mammalian sodium-leak channel is the major molecular target of substance $\mathrm{P}$ which is a potent activator of locomotion in lamprey and mouse (Smith et al., 1988; Svensson et al., 2001). It remains to be tested whether serotonin activates the sodium-leak channel.

Neuromodulatory switches are also used to switch off specific locomotory subcircuits in C. elegans. In an agar-filled Petri dish, the worm spends most of its time crawling forward, but will occasionally crawl backward either spontaneously or as an escape. The foraging behavior described previously occurs during forward locomotion but is switched off during backward locomotion (Alkema et al., 2005). A recent genetic screen for mutants that forage while moving backward revealed a critical role for the neurotransmitter tyramine. Through an elegant combination of laser ablation of identified neurons and muscles, quantitative behavioral analysis, and electrophysiology, recent studies have found that tyramine was released by the "RIM" interneuron pair apparently to hyperpolarize the "RMD" and "SMD" motorneurons and neck muscles involved in foraging through a novel tyramine-gated chloride channel (Alkema et al., 2005; Pirri et al. 2009). In C. elegans, additional members of this novel class of chloride channels were found to be gated by serotonin, dopamine, acetylcholine, and choline (Ringstad et al., 2009). These channels might serve as neuromodulatory switches to turn off specific motor programs in C. elegans.

\section{THE BIOGENIC AMINES: MECHANISMS OF BEHAVIORAL SWITCHING SHARED ACROSS ANIMAL SPECIES}

Over the course of evolution, modern gene orthologs within different animal species have been found to control behaviors that have only slightly or moderately changed from their presumed original form (e.g., circadian rhythms, as well as diverse types of serotonin-dependent learning) (Kandel, 2001; Sitaraman et al., 2008; Zheng and Sehgal, 2008). This surprising degree of conservation, not only of gene sequence but also gene function may, in part, be because such genes shared similar expression patterns in fundamentally conserved neural circuits for behavior, regardless of whether the extant behaviors are now similar or not. For example, it has been shown that homologous serotonergic neurons across species have the potential to become co-opted for the production of divergent locomotor behaviors (Newcomb and Katz, 2007). An important question that can not be adequately addressed within the scope of this article is how forms of locomotion may have

\section{REFERENCES}

Alexander, R. (1989). Optimization and gaits in the locomotion of vertebrates. Physiol. Rev. 69, 1199-1227.

Alkema, M. J., Hunter-Ensor, M., Ringstad, N., and Horvitz H. R. (2005). Tyramine functionsindependentlyof octopamine in the Caenorhabditis elegans nervous system. Neuron 46, 247-260.
Aragona, B. J., and Wang, Z. (2009). Dopamine regulation of social choice in a monogamous rodent species. Front. Behav. Neurosci. 3, 15. doi: 10.3389/neuro.08.015.2009.

Ben Arous, J., Tanizawa, Y., Rabinowitch, I., Chatenay, D., and Schafer, W. R. (2010). Automated imaging of neuronal activity in freely behaving

evolved within and between animal species. Regardless, for $C$. elegans, it is apparent that the sensory-gating of locomotion and the amines are intimately connected, especially as many of the mechanosensory neurons are themselves aminergic. In the leech, the aminergic networks are centrally located; however, it has been shown that activation of specific mechanosensory neurons important for the normal activation of swimming can excite specific serotonergic and octopaminergic neurons in the CNS (Gilchrist and Mesce 1997; Crisp and Mesce, 2006). Although we have yet to identify sensory neurons that excite centrally-located dopaminergic neurons in the leech, it is known that specific dopaminergic neurons are electrically coupled to cephalic neurons that can terminate swimming (Crisp and Mesce, 2004). Because these same descending neurons also receive sensory information from the body's mechanoreceptors (Brodfuehrer and Friesen, 1986), it could be argued that the dopaminergic network also processes sensory information.

In invertebrates and vertebrates the biogenic amines, especially serotonin, play vital roles for facilitating switching between different behaviors and for selecting specific forms of locomotory outputs (Harris-Warrick and Marder, 1991; Faulkes, 2005; Gillette, 2006; Guertin and Steuer, 2009). It is also compelling that the dopaminergic innervation of the striatum and its similar types of afferent inputs are conserved throughout evolution from the cyclostomes (e.g., lamprey) to primates (Smeets et al., 2000; Thompson et al., 2008). Thus it is not too surprising to learn that the neuromodulators released directly and indirectly by sensory neurons to adjust motor behaviors in C. elegans and the leech (e.g., serotonin, dopamine, octopamine - the analog of norepinephrine) are among the best characterized neuroactive substances known to bias higher-order decision-making processes in mammals, including humans (Aragona and Wang, 2009; Crockett, 2009). Human locomotor disorders, such as Parkinson's disease, often involve difficulty in movement initiation and switching between various motor activities; switching moods is central to our understanding and treatment of bipolar disorders and switching our attention too frequently is at the core of attention deficit hyperactive disorders. Understanding common neural strategies for locomotor decision-making among diverse animal systems may provide new ways of switching our own thinking about behavioral choice and the decisions we make - or that are made for us.

\section{ACKNOWLEDGMENTS}

We are grateful to Marty Shankland, Kathleen Klukas, Josh Puhl, Layla Young, Stephen Topper, and Andrés Vidal-Gadea for providing constructive feedback on drafts of this article. This work was supported by a grant from the National Science Foundation (IOS-0924155) (Karen A. Mesce) and funds from the University of Texas (Jonathan T. Pierce-Shimomura).

Caenorhabditis elegans. J. Neurosci. Methods 187, 229-234.

Berri, S., Boyle, J. H., Tassieri, M., Hope, I. A., and Cohen, N. (2009). Forward locomotion of the nematode C. elegans is achieved through modulation of a single gait. HFSP J. 3, 186-193.

Briggman, K. L., and Kristan, W. B. Jr. (2006). Imaging dedicated and multifunctional neural circuits generating distinct behaviors. J. Neurosci. 26, 10925-10933.

Briggman, K. L., and Kristan, W. B. Jr. (2008). Multifunctional patterngenerating circuits. Annu. Rev. Neurosci. 31, 271-294.

Brodfuehrer, P. D., and Friesen, W. O. (1986). Initiation of swimming 
activity by trigger neurons in the leech subesophageal ganglion III: sensory inputs to $\operatorname{Tr} 1$ and $\operatorname{Tr} 2$.J. Comp. Physiol. A 159, 511-519.

Cacciatore, T. W., Rozenshteyn, R., and Kristan,W.B.Jr.(2000).Kinematics and modeling of leech crawling: evidence for an oscillatory behavior produced by propagating waves of excitation. $J$. Neurosci. 20, 1643-1655.

Crisp, K. M., and Mesce, K. A. (2003). To swim or not to swim: regional effects of serotonin, octopamine and amine mixtures in the medicinal leech. J. Comp. Physiol. A Neuroethol. Sens. Neural. Behav. Physiol. 189, 461-470.

Crisp, K. M., and Mesce, K. A. (2004). A cephalic projection neuron involved in locomotion is dye coupled to the dopaminergic neural network in the medicinal leech. J. Exp. Biol. 207, 4535-4542.

Crisp, K. M., and Mesce, K. A. (2006). Beyond the central pattern generator: amine modulation of decisionmaking neural pathways descending from the brain of the medicinal leech. J. Exp. Biol. 209, 1746-1756.

Crockett,M.J.(2009). The neurochemistry of fairness: clarifying the link between serotonin and prosocial behavior. Ann. N. Y. Acad. Sci. 1167, 76-86.

de Bono, M., and Maricq, A. V. (2005). Neuronal substrates of complex behaviors in C. elegans. Annu. Rev. Neurosci. 28, 451-501.

Dunn, C. W., Hejnol, A., Matus, D. Q., Pang, K., Browne, W. E., Smith, S. A., Seaver, E., Rouse, G. W., Obst, M., and Edgecombe, G. D. (2008). Broad phylogenomic sampling improves resolution of the animal tree of life. Nature $452,745-749$.

Esch, T., Mesce, K. A., and Kristan, W. B. (2002). Evidence for sequential decision making in the medicinal leech. J. Neurosci. 22, 11045-11054.

Faulkes, Z. (2005). Mechanisms of behavioral switching. J. Comp. Physiol. A Neuroethol. Sens. Neural. Behav. Physiol. 191, 197-199.

Faumont, S., Lockery, S., Rondeau, G., and Zemek, J. (2008). Imaging a moving target. Biophotonics 15, 28-31.

Friesen, W. O., and Kristan, W. B. Jr. (2007). Leech locomotion: swimming, crawling, and decisions. Curr. Opin. Neurobiol. 17, 704-711.

Gaudry, Q., and Kristan, W. B. (2009). Behavioral choice by presynaptic inhibition of tactile sensory terminals. Nat. Neurosci. 12, 1450-1457.

Gilchrist, L. S., and Mesce, K. A. (1997). Coactivation of putative octopamine-and serotonin-containing interneurons in the medicinal leech. J. Neurophysiol. 78, 2108-2115.

Gillette, R. (2006). Evolution and function in serotonergic systems. Integr. Comp. Biol. 46, 838-846.

Gray, J., Lissmann, H. W., and Pumphrey, R. J. (1938). The mechanism of locomotion in the leech (Hirudo medicinalis Ray). J. Exp. Biol. 15, 408-430.

Guertin, P. A., and Steuer, I. (2009). Key central pattern generators of the spinal cord. J. Neurosci. Res. 87, 2399-2405.

Harris-Warrick, R. M., and Marder, E. (1991). Modulation of neural networks for behavior. Annu. Rev. Neurosci. 14, 39-57.

Kandel, E. R. (2001). The molecular biology of memory storage: a dialogue between genes and synapses. Science 294, 1030-1038.

Kiani, R., and Shadlen, M. N. (2009). Representation of confidence associated with a decision by neurons in the parietal cortex. Science 324, 759-764.

Körding, K. (2007). Decision theory: what "should" the nervous system do? Science 318, 606-610.

Korta, J., Clark, D. A., Gabel, C. V., Mahadevan, L., and Samuel, A. D. T. (2007). Mechanosensation and mechanical load modulate the locomotory gait of swimming C. elegans. J. Exp. Biol. 210, 2383-2389.

Kristan, W. B. (2008). Neuronal decision-making circuits. Curr. Biol. 18, 928-932.

Kristan, W.B. Jr., and Shaw, B. K. (1997). Population coding and behavioral choice. Curr. Opin. Neurobiol. 7 , 826-831.

Kristan, W. B., Calabrese, R. L., and Friesen, W. O. (2005). Neuronal control of leech behavior. Prog. Neurobiol. 76, 279-327.

Lu, B., Su, Y., Das, S., Wang, H., Wang, Y., Liu, J., and Ren, D. (2009). Peptide neurotransmitters activate a cation channel complex of NALCN and UNC-80. Nature 457, 741-744.

McDonald, P. W., Hardie, S. L., Jessen, T. N., Carvelli, L., Matthies, D. S., and Blakely, R. D. (2007). Vigorous motor activity in Caenorhabditis elegans requires efficient clearance of dopamine mediated by synaptic localization of the dopamine transporter DAT-1. J. Neurosci. 27, 14216-14227.

Mesce, K. A., Esch, T., and Kristan, W. B. (2008). Cellular substrates of action selection: a cluster of higher-order descending neurons shapes body posture and locomotion. J. Comp.
Physiol. A Neuroethol. Sens. Neural. Behav. Physiol. 194, 469-481.

Miller, K. G., Alfonso, A., Nguyen, M., Crowell, J. A., Johnson, C. D., and Rand, J. B. (1996). A genetic selection for Caenorhabditis elegans synaptic transmission mutants. Proc. Natl. Acad. Sci. U.S.A. 93, 12593-12598.

Newcomb, J. M., and Katz, P. S. (2007). Homologues of serotonergic central pattern generator neurons in related nudibranch molluscs with divergent behaviors. J. Comp. Physiol. A Neuroethol. Sens. Neural. Behav. Physiol. 193, 425-443.

O'Gara, B. A., Chae, H., Latham, L. B., and Friesen, W.O. (1991). Modification of leech behavior patterns by reserpineinduced amine depletion. J. Neurosci. 11, 96-110.

Pierce-Shimomura, J., Young, L., and Topper, S. (2009). Neural mechanisms for switching locomotory patterns in $C$. elegans. Abstr. Soc. Neurosci. 366, 17.

Pierce-Shimomura, J.T., Chen, B. L., Mun, J. J., Ho, R., Sarkis, R., and McIntire, S. L. (2008). Genetic analysis of crawling and swimming locomotory patterns in C. elegans. Proc. Natl. Acad. Sci. U.S.A. 105, 20982-20987.

Pirri, J. K., McPherson, A. D., Donnelly, J. L., Francis, M. M., and Alkema, M. J. (2009). A tyramine-gated chloride channel coordinates distinct motor programs of a Caenorhabditis elegans escape response. Neuron 62, 526-538.

Puhl, J. G., and Mesce, K. A. (2008). Dopamine activates the motor pattern for crawling in the medicinal leech. J. Neurosci. 28, 4192-4200.

Puhl, J. G., and Mesce, K. A. (2010). Keeping it together: mechanisms of intersegmental coordination for a flexible locomotor behavior. J. Neurosci. 30, 2373-2383.

Ringstad, N., Abe, N., and Horvitz, H. R. (2009). Ligand-gated chloride channels are receptors for biogenic amines in C. elegans. Science 325, 96-100.

Sawin, E. R., Ranganathan, R., and Horvitz, H. R. (2000). C. elegans locomotory rate is modulated by the environment through a dopaminergic pathway and by experience through a serotonergic pathway. Neuron 26, 619-631.

Sitaraman, D., Zars, M., Laferriere, H., Chen, Y. C., Sable-Smith, A., Kitamoto, T., Rottinghaus, G. E., and Zars, T. (2008). Serotonin is necessary for place memory in Drosophila. Proc. Natl. Acad. Sci. U.S.A. 105, 5579-5584.

Smeets, W.J.A.J., Marín, O., and González, A. (2000). Evolution of the basal ganglia: new perspectives through a comparative approach. J. Anat. 196, 501-517.

Smith, J. C., Feldman, J. L., and Schmidt, B. J. (1988). Neural mechanisms generating locomotion studied in mammalian brain stem-spinal cord in vitro. FASEB J. 2, 2283-2288.

Sulston, J., Dew, M., and Brenner, S. (1975). Dopaminergic neurons in the nematode Caenorhabditis elegans. J. Comp. Neurol. 163, 215-226.

Svensson, E., Grillner, S., and Parker, D. (2001). Gating and braking of shortand long-term modulatory effects by interactions between colocalized neuromodulators. J. Neurosci. 21 , 5984-5992.

Thompson, R. H., Ménard, A., Pombal, M., and Grillner, S. (2008). Forebrain dopamine depletion impairs motor behavior in lamprey. Eur. J. Neurosci. 27, 1452-1460.

Vidal-Gadea, A., Topper, S., Young, L., and Pierce-Shimomura, J. T. (2009). Neural mechanisms for switching locomotory patterns in C. elegans. Neuroscience, 366, 17. Calabrese Ronald: Faculty of 1000 Biology, 11 November, 2009. Conference 2009 October 16-21. http://f1000biology. com/article/id/1182956/evaluation

Willard, A. L. (1981). Effects of serotonin on the generation of the motor program for swimming by the medicinal leech. J. Neurosci. 1, 936-944.

Zheng, X., and Sehgal, A. (2008). Probing the relative importance of molecular oscillations in the circadian clock. Genetics 178, 1147-1155.

Conflict of Interest Statement: The authors declare that the research was conducted in the absence of any commercial or financial relationships that could be construed as a potential conflict of interest.

Received: 01 March 2010; paper pending published: 21 June 2010; accepted: 12 July 2010; published online: 30 July 2010.

Citation:Mesce KA and Pierce-Shimomura JT (2010) Shared strategies for behavioral switching: understanding how locomotor patterns are turned on and off. Front. Behav. Neurosci. 4:49. doi: 10.3389/ fnbeh.2010.00049

Copyright (๑) 2010 Mesce and Pierce-Shimomura. This is an open-access article subject to an exclusive license agreement between the authors and the Frontiers Research Foundation, which permits unrestricted use, distribution, and reproduction in any medium, provided the original authors and source are credited. 